\title{
The Effect of Percutaneous Coronary Intervention on Left Ventricular Diastolic Dysfunction in Patients with Coronary Artery Disease Assessed by Strain Rate Imaging
}

\author{
Fariba Bayat ${ }^{1}$, Elham Farahani ${ }^{2 *}$, Habibollah Saadat ${ }^{1}$ \\ ${ }^{1}$ Shahid Beheshti University of Medical Sciences, Tehran, Iran \\ ${ }^{2}$ Cardiovascular Research Center, Modarres Hospital, Shahid Beheshti University of Medical Sciences, Tehran, \\ Iran \\ Email: ${ }^{\text {farahani13025@gmail.com }}$
}

Received 23 July 2014; revised 9 September 2014; accepted 24 September 2014

Copyright (C) 2014 by authors and Scientific Research Publishing Inc.

This work is licensed under the Creative Commons Attribution International License (CC BY). http://creativecommons.org/licenses/by/4.0/

(c) $\underset{\mathrm{EY}}{\mathrm{E}}$ Open Access

\section{Abstract}

Introduction: Coronary artery disease (CAD) is the most common cause for left ventricular dysfunction. Unfortunately, the treatment strategies of regional myocardial diastolic dysfunction in patients with CAD have not been well characterized and benefit of percutaneous coronary intervention (PCI) as a treatment strategy is not clear. So the present study aimed to assess the effects of PCI on regional and global left ventricular diastolic dysfunction in patients with CAD assessed by strain rate (SR) imaging. Methods: Thirty adult symptomatic patients with coronary artery disease that underwent coronary angiography and candidate for PCI on left anterior descending artery were enrolled to our study. Echocardiographic findings and early diastolic SR were measured before and 48 hours after PCI. Results: Mean age of the patients was 59.9 \pm 8.3 years. Most of the left ventricular diastolic parameters showed significant difference before and after elective PCI; while mitral E velocity, DT, E/A and pulmonary vein flow before and after PCI did not show significant difference assessed by statically test. Also before PCI, mean (SD) of peak early diastolic SR in ischemic regions $(1.89 \pm 0.22)$ was smaller than of non-ischemic regions $(2.53 \pm 0.26)$ while after PCI this parameter became similar in ischemic regions $(2.55 \pm 0.27)$ and non-ischemic regions $(2.55 \pm 0.26)$. Conclusion: Most of the left ventricular diastolic parameters improved after PCI in CAD patients. Also regional myocardial relaxation as measured by peak early diastolic $S R\left(E_{S R}\right)$ in the ischemic segments improved significantly compared with that in non-ischemic segments.

\footnotetext{
${ }^{*}$ Corresponding author.
} 


\section{Keywords}

\section{Strain Rate, Percutaneous Coronary Intervention, Diastolic Function}

\section{Introduction}

Left ventricular diastolic dysfunction is an important clinical parameter which is associated with a substantial risk of subsequent development of congestive heart failure and reduced survival of patients similar to what happens in systolic dysfunction [1]. The prevalence of diastolic dysfunction is more than systolic dysfunction and is increased with aging [2]. This point is more important when we know the rate of mortality in patients with asymptomatic diastolic dysfunction is fivefold in comparison with general population [3].

Evaluation of regional left ventricular diastolic function could be a good strategy to identify myocardial regions with impaired coronary artery flow and reduced myocardial perfusion.

Echocardiographic Doppler tissue strain rate (SR) imaging is a new method of echocardiography that yield information regarding regional diastolic function in coronary artery disease (CAD) patients by using tissue velocity data to calculate velocity gradients between two distinct points along the ultrasound beam.

Unfortunately, the treatment strategies of regional myocardial diastolic dysfunction in patients with CAD have not been well characterized [4] and literature is very limited in regional diastolic dysfunction in comparison with systolic dysfunction.

Percutaneous coronary intervention (PCI) is reperfusion strategy in improvement of global and regional myocardial function and provides a feasible advance in management of quality of life [5].

However, the widespread use and recurrent improvements of this method have resulted in a significant enhance in survival rate compared with pharmacological reperfusion therapy [6] but results of echocardiographic assessment of systolic and diastolic ventricular function following successful elective PCI have been contradictory and confusing [7]-[11].

So the present study aimed to assess the effects of PCI on regional and global left ventricular diastolic dysfunction in patients with CAD by using SR imaging.

\section{Methods}

\subsection{Study Population}

Thirty adult symptomatic patients who were known cases of CAD that underwent coronary angiography and had significant lesion on LAD (candidate for PCI) with mild left ventricular diastolic dysfunction (LV ejection fraction: $53.5 \% \pm 5.7 \%$; range $45 \%$ - 68\%) and presented to the cardiology department of Shahid Modarres University Hospital in Tehran-Iran were enrolled. Patients with single vessel CAD based on coronary angiography (LAD stenosis over than 70\%) and echocardiography (EF over than 40\%) were eligible for inclusion. Exclusion criteria included previous history of STEMI, metabolic problems such as diabetes and thyroid disease, creatinine > 2, heart block, interventricular septal hypertrophy, pulmonary HTN, atrial fibrillation, significant valvular disease, hypertrophic or idiopathic restrictive cardiomyopathy or inability to consent for the study. Demographic variables and CAD risk factors are presented in Table 1.

All patients were candidates for PCI on left anterior descending artery via femoral approach after diagnostic angiography. All echocardiographic examinations were performed by an expert cardiologist in all patients.

\subsection{Coronary Angiography and Percutaneous Coronary Intervention (PCI)}

Coronary diagnostic angiography was performed and minimal lumen and reference diameters were determined and the degree of coronary diameter stenosis was calculated from at least two views.

After diagnostic angiography PCI was performed in the usual manner with balloon catheters and a 0.014-inch, 15-MHz, Doppler-tipped angioplasty guidewire (Flo-Wire, Cardiometrics). This was performed after the administration of additional heparin to maintain the activated clotting time at 300 seconds. Also for all patients aspirin (ASA) $325 \mathrm{mg}$ as well as clopidogrel (Plavix) $600 \mathrm{mg}$ before PCI were administered. PCI was considered successful if the final stenosis was reduced to lower than $20 \%$. 
Table 1. Demographic variables and coronary artery disease (CAD) risk factors.

\begin{tabular}{cc}
\hline Variable & No. or mean (SD) \\
\hline Age, year, mean $( \pm$ SD) & $59.9 \pm 8.3$ \\
Gender, $\mathbf{M} / \mathbf{F}$ & $20 / 10$ \\
& \\
CAD risk factors & 21 \\
Hypertension & 23 \\
Hyperlipidemia & 0 (exclusion criteria) \\
Diabetes mellitus & 20 \\
Cigarette smoking & 11 \\
Positive family history of CAD & \\
Body mass index $\left(\mathbf{k g} / \mathbf{m}^{2}\right)$, mean $( \pm$ SD) & $32 \pm 4.1$ \\
\hline
\end{tabular}

After the PCI, the stented patients were maintained on a regimen of ASA 325 mg daily and Plavix 75 mg bid for 2 days and $75 \mathrm{mg}$ daily after that.

\subsection{Echocardiography}

An echocardiographic examination was performed at baseline within 24 hours before PCI and were repeated 48 hours thereafter using a digital ultrasound system (Ultasomd Vivid 7, General Motor Cor., USA) with a $2.5 \mathrm{MHz}$ phased-array transducer. Standard views, including the parasternal long-axis, short-axis at the papillary muscle level, and apical 4-chamber and 2-chamber views were recorded. We then determined left diastolic function parameters such as deceleration time (DT) (ms), isovolumic relaxation time (IVRT) (ms), peak transmitral early diastolic flow velocity (E wave), peak transmitral late diastolic flow velocity (A wave), mitral E/A ratio and pulmonary veins flow.

\subsection{Doppler Tissue and Strain Rate Imaging}

Real-time 2-dimensional color-coded tissue Doppler data were recorded from the interventricular septum using an apical long-axis, 4-chamber and 2-chamber view during brief held-expiration. The image sector was kept as narrow (30 degree) as possible to achieve frame rates of at least higher than 200 frames per second with realtime display of strain rate (SR) color images (Figure 1). Recordings were stored digitally and analyzed off-line with software (EchoPAC PC; GE Vingmed Ultrasound AS, Horten, Norway). Sample volumes were placed in the inner half of the myocardium on the basal, mid, and apical portions of LV at the anteroseptal, anterior, lateral, posterior, inferior, and posteroseptal walls in the apical views. Thus, the LV was divided into 16 segments (Figure 1). Of all segments, segments that received supply from LAD were considered as ischemic segments and other segments were considered as non-ischemic segments. Early diastolic SR was measured in all ischemic and non-ischemic segments before and 48 hours after PCI.

The SR imaging was equal to the spatial myocardial velocity gradient expressed by the equation:

$$
S R=(v[r]-v[r+\Delta r]) / \Delta r
$$

and is measured in $\mathrm{s}^{-1}(1 / \mathrm{s})$, where $v$ is the longitudinal velocity component, $r$ is the distance along the beam, and delta $r$ is the small offset between the 2 points. An offset (sample volume of SR) of delta $r=9.2 \mathrm{~mm}$ was used in all studies.

Finally, peak myocardial early diastolic flow velocity ( $E^{\prime}$ wave) and $E / E^{\prime}$ in the medial annulus were measured.

\subsection{Ethical Approval}

The study was approved 2012 by the ethics committee of the university before its initiation, and the protocols used conformed to the ethical guidelines of the 1975 Helsinki Declaration. 


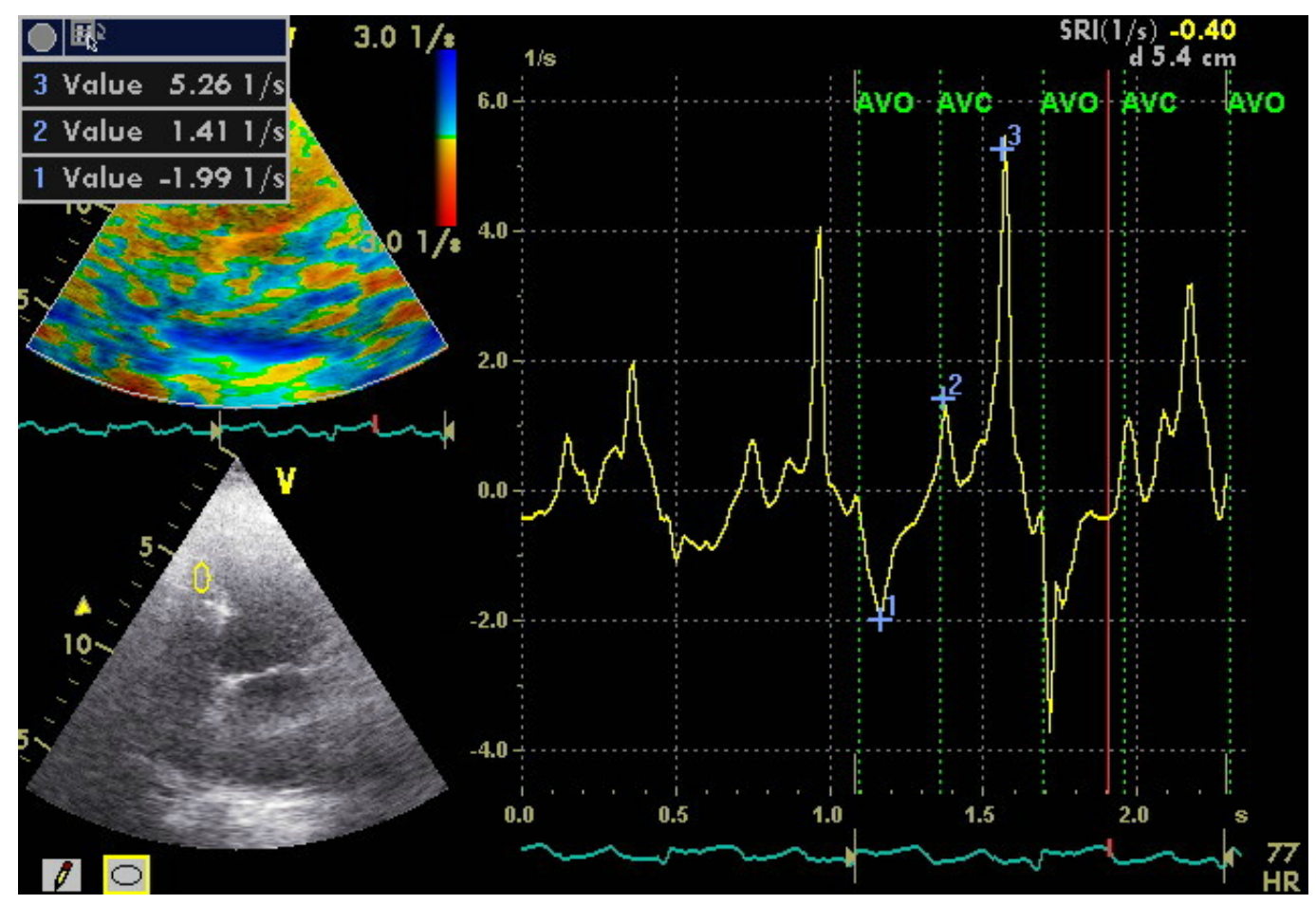

Figure 1. Example of strain rate (SR) profile. Profile shows one negative (1) and two positive waves (2 \& 3) during systole and diastole, respectively. Peak early diastolic SR (2) was measured.

\subsection{Statistical Analysis}

The statistical evaluation was performed by computer analysis with SPSS Software (Statistical Package for the Social Sciences, version 16.0, SPSS Inc., Chicago, Ill, USA). Student's paired $t$ test, McNemar exact test and covariance test to calculate adjusted $\mathrm{P}$ value were used, where appropriate, for comparing clinical data between results before and after PCI. Continuous data were demonstrated as mean \pm standard deviation. $P$-Value less than 0.05 were considered significant.

\section{Results}

All 30 patients consisting of 20 male and 10 female (mean age, $59.9 \pm 8.3$; range 43 - 79) had a mild degree of diastolic dysfunction, PCI was performed successfully in all of them and no complication was observed during hospital stay.

Most of the left ventricular diastolic parameters showed significant difference before and after elective PCI; while mitral E velocity, DT, E/A and pulmonary vein flow before and after PCI did not show any significant differences by using statically test. The echocardiographic measurements before and after PCI are presented in (Table 2).

Strain rate imaging findings showed significant increase in mean peak early diastolic SR of ischemic regions after PCI; while comparison of mean peak early diastolic SR before and after PCI in non-ischemic regions was not statistically significant (Table 3). Also before PCI, mean (SD) of peak early diastolic SR in ischemic regions $(1.89 \pm 0.22)$ was smaller than of non-ischemic regions $(2.53 \pm 0.26)$ while after PCI this parameter was becoming similar in ischemic regions (2.55 \pm 0.27$)$ and non-ischemic regions $(2.55 \pm 0.26)$.

\section{Discussion}

Left ventricular diastolic dysfunction has been reported in many patients with CAD [12]-[18] and although most frequently it is diagnosed as a preclinical disease, it constitutes a predictor of all-cause mortality [19] [20].

Thus evaluation of left ventricular diastolic function could provide valuable data to determine condition of heart to prevent heart failure and preserve suitable EF. Echocardiographic Doppler tissue SR imaging is a new 
Table 2. Echocardiographic findings in patients before and after PCI.

\begin{tabular}{cccc}
\hline Parameters & Before PCI & After PCI & $\begin{array}{c}\text { Adjusted } \\
\boldsymbol{P} \text {-value }\end{array}$ \\
\hline Mitral E velocity (cm/s) & $53.7 \pm 10$ & $57.8 \pm 10.5$ & 0.11 \\
A (cm/s) & $72.6 \pm 11.3$ & $68.6 \pm 10.9$ & $0.03^{*}$ \\
E' $^{\prime}(\mathbf{c m} / \mathbf{s})$ & $6.7 \pm 1.1$ & $8.6 \pm 1.1$ & $0.02^{*}$ \\
E/E' & $8.1 \pm 1$ & $6.8 \pm 1.2$ & $0.04^{*}$ \\
IVRT (ms) & $119.4 \pm 27.8$ & $99 \pm 17.7$ & $0.03^{*}$ \\
DT (ms) & $264.8 \pm 15.4$ & $253.4 \pm 20.6$ & 0.36 \\
S > D & 25 & 27 & \\
PV flow $<$ D & 5 & 3 & 0.52 \\
E/A & $0.72 \pm 0.09$ & $0.84 \pm 0.12$ & 0.61 \\
\hline
\end{tabular}

* $(P$-Value less than 0.05 were considered significant).

Table 3. Early diastolic Strain rate imaging in patients before and after PCI.

\begin{tabular}{cccc}
\hline Parameters & Before PCI & After PCI & $\begin{array}{c}\text { Adjusted } \\
\text { P-value }\end{array}$ \\
\hline Ischemic region $\left(\mathbf{s}^{-\mathbf{1}}\right)$ & $1.89 \pm 0.22$ & $2.55 \pm 0.27$ & $0.01^{*}$ \\
Non-ischemic region $\left(\mathbf{s}^{-\mathbf{1}}\right)$ & $2.53 \pm 0.26$ & $2.55 \pm 0.26$ & 0.26 \\
\hline
\end{tabular}

* $(P$-Value less than 0.05 were considered significant).

means of providing information regarding regional myocardial function by using tissue velocity gradients between two distinct points.

Several studies have demonstrated that this method is an appropriate tool for evaluating regional myocardial function in diastole and systole and provides significant parameters such as IVRT, the interval from the peak of $(E)$ velocity to its extrapolation to baseline (DT), E/A ratio and etc. to assess diastolic dysfunction [21]-[24].

Authors believe that some indexes are not accurate to assess LV diastolic function, principally in patients with normal EF [25]-[27]. On the other hand, some tissue Doppler parameters such as the ratios of $E / E^{\prime}$ and $E^{\prime} / A^{\prime}$ have been reported to be sensitive enough in the assessment of the LV diastolic function although the $E / E^{\prime}$ ratio has greater specificity compared to $E^{\prime} / A^{\prime}$ ratio in detecting diastolic dysfunction [27] [28].

We used peak early diastolic SR $\left(E_{S R}\right)$ to represent the maximal velocity gradient of longitudinal wall lengthening in early diastole, and $E_{S R}$ is thought to be closely related to regional myocardial relaxation. Therefore, the finding that the reduced $E_{S R}$ in the ischemic segments was increased after successful PCI suggested that impaired regional myocardial relaxation was improved in ischemic segments with PCI.

Our study demonstrated improved global and regional LV early diastolic function after PCI except mitral E velocity, DT, E/A ratio and pulmonary vein flow. In a similar study, Hashemi et al. [11] evaluated diastolic echocardiographic findings before PCI, 48 hours and 3 months after PCI in 30 patients with EF of more than $40 \%$ scheduled for elective PCI. Except mitral E/A ratio and mitral E' septal velocity all of the left ventricular diastolic parameters showed no significant improvement after 48 hours. Of course Hashemi et al. provided significant improvement in Mitral $A$ velocity moreover two previous parameter after 3 month. Tanaka et al. [1] investigated the effects of PCI on global and regional left ventricular diastolic function, using (SR) imaging in 27 patients and finally concluded that Mitral $E$ velocity, Mitral $A$ velocity, DT and $E / A$ did not reveal significant improvement after PCI.

On the other hand regional myocardial relaxation as measured by $E_{S R}$ in the ischemic segments. Our results showed that mean SR in ischemic regions before and after PCI was significantly different and this parameter after PCI showed significant increase.

Utilizing SR imaging Tanaka et al. [1] in agreement with our study showed that myocardial diastolic dysfunction improved in patients with CAD after PCI. They noted that PCI resulted in a significant increase in $E_{S R}$ in the ischemic but not in the non-ischemic, segments. The peak early diastolic trans-mitral flow velocities after PCI 
improved in patients with greater extent of improvement of $E_{S R}$ in the ischemic segments. These findings suggest that the improvement in left ventricular early diastolic filling after PCI may be associated with the degree of improvement in impaired regional myocardial relaxation.

Derumeaux et al. [29] evaluated left ventricular isovolumic relaxation blood flow as Doppler-echocardiographic parameter for the diagnosis of acute cardiac rejection and finally concluded that regional diastolic function was impaired during PCI and improved in the early period after successful PCI.

Nozari et al. [30] in another study investigated the effect of PCI on left ventricular (LV) systolic and diastolic function. The authors in this study measured echocardiographic findings of 115 patients with CAD before PCI, the day after and 3 - 6 months later. In this study, diastolic dysfunction was mild to moderate before PCI, which in 74\% (86 patients) improved to mild dysfunction the day after PCI but not changed 3 - 6 months later.

Sang Jin Ha et al. evaluated 22 patients with stable angina pectoris with angiographically significant coronary artery stenosis but normal wall motion and showed PCI caused a significant increase in peak systolic strain and peak early diastolic strain rate but not peak systolic strain rate and peak late diastolic strain rate in the ischemic segments [31].

Furthermore, our results showed that regional myocardial relaxation in the ischemic segments significantly improved after PCI. In addition, this study found that the improved global LV early diastolic filling after PCI was associated with the degree to which impaired regional myocardial relaxation improved in the ischemic segments. Improvement in myocardial relaxation in CAD caused by PCI and that regional myocardial relaxation are associated with the improvement in dynamics of global LV early diastolic filling. According to this study, regional diastolic SR significantly was improved in segments of LAD territory that is a clue for diagnosis of CAD in asymptomatic patients and specially determination of type of vessel involvement in CAD patients before coronary angiography. However more studies requisiteness to confirm these justifications. All improvements observed in this study were more significant in comparison to similar previous studies in the literature. This discrepancy may be difference in basic ventricular diastolic dysfunction and LV ejection fraction before PCI, global condition of the patients and degree and type of coronary artery stenosis on the basis of angiography.

\section{Limitation}

Overall the Echocardiographic Doppler tissue SR imaging has a number of potential pitfalls. The first is Signal noise, avoid aliasing on TVI signal and track sample volume to left ventricular wall to avoid cavity signal solve this problem. The second limitation relates to underestimation, this problem correct with using high frame rate. Another limitation is angle dependency, align axis of movement with scan line and narrow sector decrease this problem, another limitation is respiratory drift that can solve with acquire pictures in end-expiration [13].

Our results showed more improvement in results of PCI in comparison with similar study. A limitation to the current study could have been the relatively small number of patients evaluated. Moreover, early assessment of myocardial performance without any follow up could be another limitation of this study. The final shortcoming may have been the fact that we did not consider clinical improvement along with echocardiographic parameters. To our knowledge, this study is, however, the first to cover a wide range of echocardiographic indices for postPCI functional assessment in patients with stable CAD.

\section{Conclusion}

Most of the Left ventricular diastolic parameters improved after PCI in CAD patients. Also regional myocardial relaxation as measured by $E_{S R}$ in the ischemic segments improved significantly compared with that in nonischemic segments.

\section{Conflict of Interest}

None declared.

\section{References}

[1] Tanaka, H., Kawai, H., Tatsumi, K., et al. (2006) Improved Regional Myocardial Diastolic Function Assessed by Strain Rate Imaging in Patients with Coronary Artery Disease Undergoing Percutaneous Coronary Intervention. Journal of the American Society of Echocardiography, 19, 756-762. http://dx.doi.org/10.1016/j.echo.2006.01.008 
[2] Fischer, M., Baessler, A., Hense, H.W., et al. (2003) Prevalence of Left Ventricular Diastolic Dysfunction in the Community. Results from a Doppler Echocardiographic-Based Survey of a Population Sample. European Heart Journal, 24, 320-328. http://dx.doi.org/10.1016/S0195-668X(02)00428-1

[3] Betocchi, S. and Hess, O. (2000) LV Hypertrophy and Diastolic Heart Failure. Heart Failure Reviews, 5, 333-336. http://dx.doi.org/10.1023/A:1026559113044

[4] Hunt, S.A., Baker, D.W., Chin, M.H., Cinquegrani, M.P., Feldman, A.M., Francis, G.S., Ganiats, T.G., Goldstein, S., Gregoratos, G., Jessup, M.L., Noble, R.J., Packer, M., Silver, M.A., Stevenson, L.W., Gibbons, R.J., Antman, E.M., Alpert, J.S., Faxon, D.P., Fuster, V., Gregoratos, G., Jacobs, A.K., Hiratzka, L.F., Russell, R.O., Smith Jr., S.C., American College of Cardiology/American Heart Association Task Force on Practice Guidelines (Committee to Revise the 1995 Guidelines for the Evaluation and Management of Heart Failure); International Society for Heart and Lung Transplantation; Heart Failure Society of America (2001) ACC/AHA Guidelines for the Evaluation and Management of Chronic Heart Failure in the Adult: Executive Summary a Report of the American College of Cardiology/American Heart Association Task Force on Practice Guidelines (Committee to Revise the 1995 Guidelines for the Evaluation and Management of Heart Failure): Developed in Collaboration with the International Society for Heart and Lung Transplantation; Endorsed by the Heart Failure Society of America. Circulation, 104, 2996-3007. http://dx.doi.org/10.1161/hc4901.102568

[5] Taylor, R.S., Brown, A., Ebrahim, S., et al. (2004) Exercise-Based Rehabilitation for Patients with Coronary Heart Disease: Systematic Review and Meta-Analysis of Randomized Controlled Trials. American Journal of Medicine, 116, 682-692. http://dx.doi.org/10.1016/j.amjmed.2004.01.009

[6] Andersen, H.R., Nielsen, T.T., Rasmussen, K., et al. (2003) A Comparison of Coronary Angioplasty with Fibrinolytic Therapy in Acute Myocardial Infarction. The New England Journal of Medicine, 49, 733-742. http://dx.doi.org/10.1056/NEJMoa025142

[7] Çaylý, M., Usal, A., Demir, M., et al. (2007) The Effect of Successful Elective Percutaneous Coronary Intervention on Left Ventricular Functions Assessed with Tissue Doppler Imaging Method. Tanýsal ve Giripimsel Radyoloji Dergisi, 11, 146-150.

[8] Tumuklu, M., Kayikcioglu, M., Aliyev, E., et al. (2003) Evaluation of Early Alterations in Transmitral Diastolic Flow and Tissue Doppler Findings of the Basal Segments of Both Ventricles in Early Period after Coronary Angioplasty. Anadolu Kardiyoloji Dergisi, 3, 16-23.

[9] Rashid, H., Abdel-Moniem, A., Email, S. and El-Batran, M. (2012) Evaluation of Myocardial Function in Patients with Chronic Stable Angina and Apparent Normal Ventricular Function (Tissue Doppler Study before and after PCI). Heart Mirror Journal, 6, 141-147.

[10] Hueb, J.C., Hepner, A., Hueb, R.A.S, Okoshi, K., Zanati, S.G. and Matsubara, B.B. (2011) Percutaneous Coronary Intervention Improves Left Ventricular Diastolic Function in Patients with Stable Angina? Revista Brasileira de Ecocardiografia e Imagem Cardiovascular, 24, 29-34.

[11] Hashemi, S.R., Motamedi, M., Khani, M., Hekmat, M., Gachkar, L. and Rezaeefar, A. (2010) Evaluation of the Effect of Elective Percutaneous Coronary Intervention as a Treatment Method on the Left Ventricular Diastolic Dysfunction in Patients with Coronary Artery Disease. Journal of Tehran Heart Center, 5, 194-198.

[12] Ishii, K., Suyama, T., Imai, M., Maenaka, M., Yamanaka, A., Makino, Y., et al. (2009) Abnormal Regional Left Ventricular Systolic and Diastolic Function in Patients with Coronary Artery Disease Undergoing Percutaneous Coronary Intervention. Journal of the American College of Cardiology, 54, 1589-1597. http://dx.doi.org/10.1016/j.jacc.2009.06.030

[13] Marwick, T.H. (2006) Measurement of Strain and Strain Rate by Echocardiography: Ready for Prime Time? Journal of the American College of Cardiology, 47, 1313-1327. http://dx.doi.org/10.1016/j.jacc.2005.11.063

[14] Djordjevic-Dikic, A. (2011) Echocardiographic Measurements Promising for Predicting Improvement after Elective PCI. Journal of the American Society of Echocardiography, 24, 573-581.

[15] Sampat, U., Prasad, A., Mahmud, E., Ilapakurti, M., Palakodeti, V. and DeMaria, A.N. (2011) Evidence of Ischemic Memory from Strain Rate Analysis of Diastolic Function during/after Percutaneous Coronary Intervention. Circulation, 124, A16710.

[16] Manda, A.K., Chowdhury, A.H.K., Choudhury, A.K., Monwarul Islam, A.K.M. and Guha, B. (2012) Echocardiographic Evaluation of Left Ventricular Diastolic Function after Percutaneous Coronary Intervention in Patients with Coronary Artery Disease. Cardiovascular Journal, 4, 127-131.

[17] Shemirani, H., Karimi, K. and Madad, R. (2012) Evaluation of Early Alterations in Transmitral Diastolic Flow and Tissue Doppler Imaging Findings of the Septal and Lateral Segments of the Left Ventricle in the Early Period after Coronary Angioplasty. ARYA Atherosclerosis Journal, 7, S13-S16.

[18] Šipić, T., Štambuk, K., Trbović, A., Kapov-Sviličić, K., Szavits-Nossan, J. and Bernat, R. (2013) Echocardiographic Assessment of Revascularization Completeness Impact on Diastolic Dysfunction in Ischemic Heart Disease. Collegium 
Antropologicum, 37, 1299-1305.

[19] Abhayaratna, W.P., Srikusalanukul, W. and Budge, M.M. (2008) Aortic Stiffness for the Detection of Preclinical Left Ventricular Diastolic Dysfunction: Pulse Wave Velocity versus Pulse Pressure. Journal of Hypertension, 26, 758-764. http://dx.doi.org/10.1097/HJH.0b013e3282f55038

[20] Redfield, M.M., Jacobsen, S.J., Burnett Jr., J.C., Mahoney, D.W., Bailey, K.R. and Rodeheffer, R.J. (2003) Burden of Systolic and Diastolic Ventricular Dysfunction in the Community: Appreciating the Scope of the Heart Failure Epidemic. JAMA, 289, 194-202. http://dx.doi.org/10.1001/jama.289.2.194

[21] Voigt, J.U., Lindenmeier, G., Werner, D., Flachskampf, F.A., Nixdorff, U., Hatle, L., Sutherland, G.R. and Daniel, W.G. (2002) Strain Rate Imaging for the Assessment of Preload-Dependent Changes in Regional Left Ventricular Diastolic Longitudinal Function. Journal of the American Society of Echocardiography, 15, 13-19. http://dx.doi.org/10.1067/mje.2002.116536

[22] Stoylen, A., Slordahl, S., Skjelvan, G.K., Heimdal, A. and Skjaerpe, T. (2001) Strain Rate Imaging in Normal and Reduced Diastolic Function: Comparison with Pulsed Doppler Tissue Imaging of the Mitral Annulus. Journal of the American Society of Echocardiography, 14, 264-274. http://dx.doi.org/10.1067/mje.2001.110375

[23] Hashimoto, I., Li, X., Hejmadi Bhat, A., Jones, M., Zetts, A.D. and Sahn, D.J. (2003) Myocardial Strain Rate Is a Superior Method for Evaluation of Left Ventricular Subendocardial Function Compared with Tissue Doppler Imaging. Journal of the American College of Cardiology, 42, 1574-1583. http://dx.doi.org/10.1016/j.jacc.2003.05.002

[24] Cosson, S. and Kevorkian, J.P. (2003) Left Ventricular Diastolic Dysfunction: An Early Sign of Diabetic Cardiomyopathy? Diabetes \& Metabolism, 29, 455-466. http://dx.doi.org/10.1016/S1262-3636(07)70059-9

[25] Diller, G.P., Wasan, B.S., Thom, S.A., Foale, R.A., Hughes, A.D., Francis, D.P. and Mayet, J. (2009) Evidence of Improved Regional Myocardial Function in Patients with Chronic Stable Angina and Apparent Normal Ventricular Function-A Tissue Doppler Study before and after Percutaneous Coronary Intervention. Journal of the American Society of Echocardiography, 22, 177-182. http://dx.doi.org/10.1016/j.echo.2008.10.018

[26] Yamamoto, K., Nishimura, R.A., Chaliki, H.P., Appleton, C.P., Holmes Jr., D.R. and Redfield, M.M. (1997) Dermination of Left Ventricular Filling Pressure by Doppler Echocardiography in Patients with Coronary Artery Disease: Critical Role of Left Ventricular Systolic Function. Journal of the American College of Cardiology, 30, 1819-1826. http://dx.doi.org/10.1016/S0735-1097(97)00390-2

[27] Ommen, S.R., Nishimura, R.A., Appleton, C.P., Miller, F.A., Oh, J.K., Redfield, M.M., et al. (2000) Clinical Utility of Doppler Echocardiography and Tissue Doppler Imaging in the Estimation of Left Ventricular Filling Pressures: A Comparative Simultaneous Doppler-Catheterization Study. Circulation, 102, 1788-1794. http://dx.doi.org/10.1161/01.CIR.102.15.1788

[28] Kasner, M., Westermann, D., Steendijk, P., Gaub, R., Wilkenshoff, U., Weitmann, K., et al. (2007) Utility of Doppler Echocardiography and Tissue Doppler Imaging in the Estimation of Diastolic Function in Heart Failure with Normal Ejection Fraction: A Comparative Doppler-Conductance Catheterization Study. Circulation, 116, 637-647. http://dx.doi.org/10.1161/CIRCULATIONAHA.106.661983

[29] Derumeaux, G., Mouton-Schleifer, D., Redonnet, M., Cribier, A., Soyer, R. and Letac, B. (1998) Blood Flow in the Isovolumetric Relaxation Phase in Heart Transplant Patients. Its Use in the Noninvasive Diagnosis of Acute Rejection. Archives des Maladies du Coeur et des Vaisseaux, 91, 731-738.

[30] Nozari, Y., Oskouei, N.J. and Khazaeipour, Z. (2012) Effect of Elective Percutaneous Coronary Intervention on Left Ventricular Function in Patients with Coronary Artery Disease. Acta Medica Iranica, 50, 26-30.

[31] Ha, S.J., Kim, S.H., Kim, J.B., Kim, S.J., Kim, W. and Kim, W. (2012) Improvement in Regional Myocardial Function Assessed by Strain Rate Imaging in Patients who had Coronary Artery Disease but Normal Wall Motion Undergoing Percutaneous Coronary Intervention. Circulation, 126, A17807. 
Scientific Research Publishing (SCIRP) is one of the largest Open Access journal publishers. It is currently publishing more than 200 open access, online, peer-reviewed journals covering a wide range of academic disciplines. SCIRP serves the worldwide academic communities and contributes to the progress and application of science with its publication.

Other selected journals from SCIRP are listed as below. Submit your manuscript to us via either submit@scirp.org or Online Submission Portal.

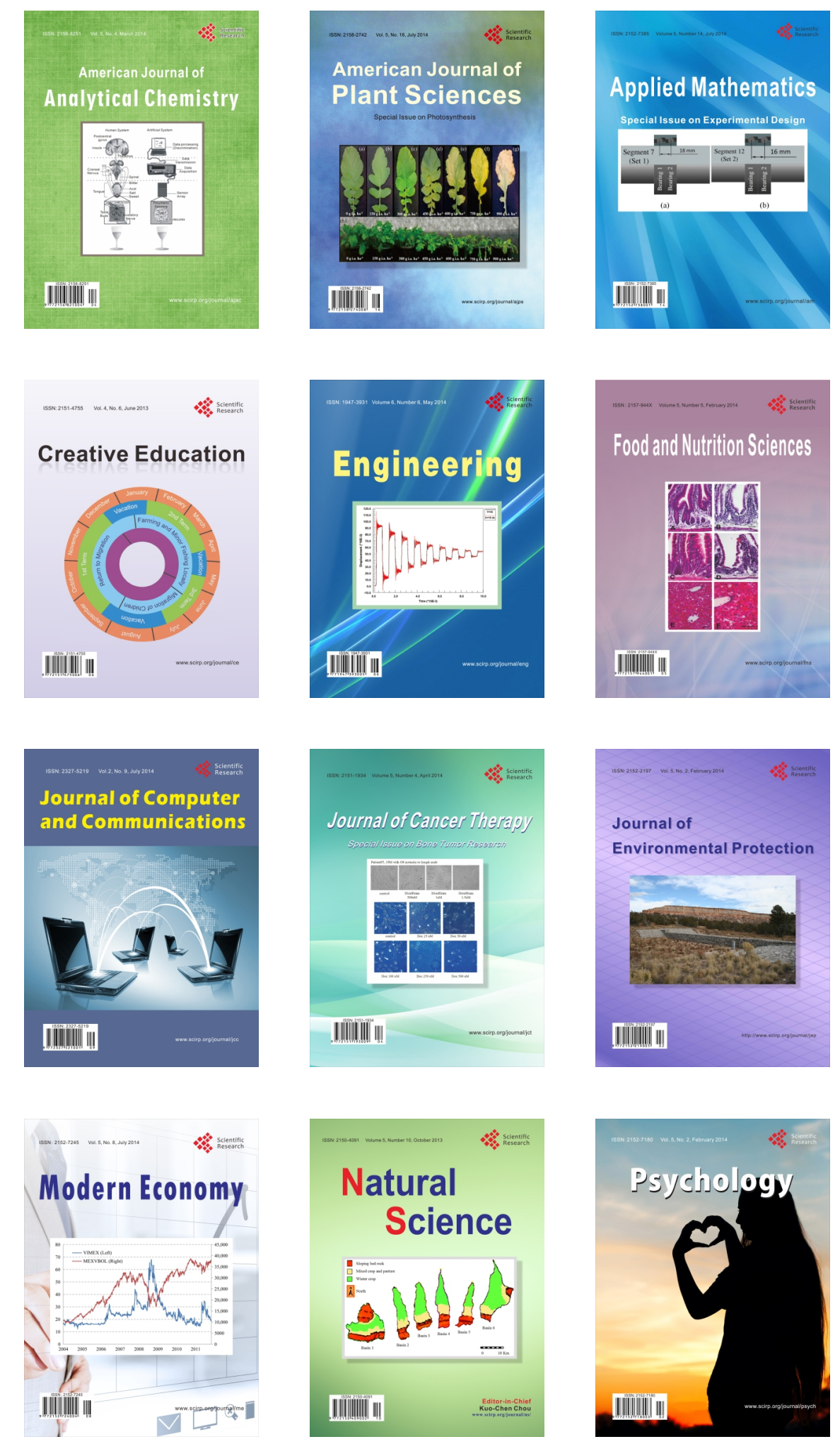